\title{
BEAM TEST RESULTS WITH THE ISAC 35 MHZ RFQ
}

\author{
R.E. Laxdal, R.A. Baartman, L. Root
}

\begin{abstract}
The ISAC $35 \mathrm{MHz}$ split-ring RFQ is designed to accelerate ions of $A / q \leq 30$ from $2 \mathrm{keV} / \mathrm{u}$ to $150 \mathrm{keV} / \mathrm{u}$ in cw mode at a constant synchronous phase of $-25^{\circ}$. Beam tests with 7 of the 19 split rings installed ( $E_{\text {fin }}=0.55 \mathrm{keV} / \mathrm{u}$ ) have recently been completed. The tests demonstrated that a constant synchronous phase RFQ with external buncher works splendidly for low beta, low intensity applications. One peculiar aspect of the RFQ is that the longitudinal acceptance is larger for beams injected off-center. The test set-up and results of both the measurements and the simulations are presented and discussed.
\end{abstract}

\section{INTRODUCTION}

A radioactive ion beam facility with on-line source and linear post-accelerator is being built at TRIUMF[1]. The accelerator chain includes a $35 \mathrm{MHz}$ split-ring RFQ, operating $\mathrm{cw}$, to accelerate beams of $A / q \leq 30$ from $2 \mathrm{keV} / \mathrm{u}$ to $150 \mathrm{keV} / \mathrm{u}$ [2]. The final RFQ electrodes will span $7.6 \mathrm{~m}$ with 19 modules each consisting of one ring and $40 \mathrm{~cm}$ of electrodes. The gross specifications include a bore radius of $r_{0}=7.4 \mathrm{~mm}$, and a maximum inter-vane voltage of $74 \mathrm{kV}$. A unique feature of the design is the constant synchronous phase of $-25^{\circ}[3]$. The buncher and shaper sections of the RFQ have been eliminated in favour of a four-harmonic sawtooth pre-buncher located $\sim 5 \mathrm{~m}$ upstream of the RFQ in the low energy beam transport (LEBT). This shortens the RFQ and yields a smaller longitudinal emittance at the expense of a slightly lower beam capture. The pre-buncher operates at a fundamental frequency of $11.7 \mathrm{MHz}$, one third the frequency of the RFQ. Presently three harmonics are operational on the pre-buncher resulting in a predicted capture efficiency of $80 \%$.

It was decided to proceed with a two-stage installation. We have installed and tested with beam the first seven rings of the RFQ. Installation of the remaining twelve rings is now in progress.

\section{THE TEST SET-UP}

A schematic of the test set-up is shown in Fig. 1. An rf short is placed after the seventh module to confine the rf fields. Eight electrostatic quadrupoles are used to transport the beam to a diagnostic station downstream of the RFQ tank. A Faraday cup and profile monitor assembly are placed just after the RFQ short and again in the middle of the electrostatic transport after the fourth quadrupole. The quadrupoles when tuned act as an energy filter; the unaccelerated beam is lost in the first few quadrupoles. Therefore the transmission difference between the first Faraday cup and the second marks the capture efficiency of the RFQ.

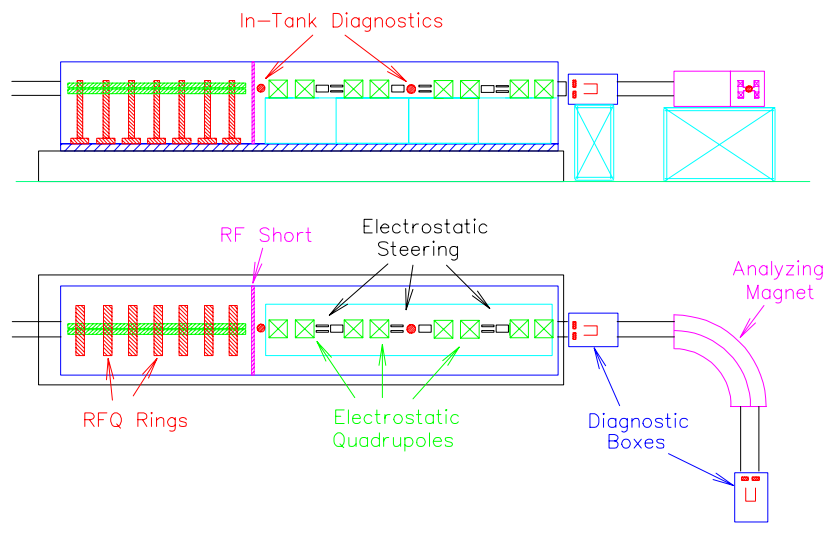

Figure 1: Schematic diagram of the test set-up.

The diagnostic station shown in Fig. 1 includes a Faraday cup, profile monitor and a spectrometer consisting of an object and image slit and a 90 degree bending magnet. A slit and harp transverse emittance rig is positioned upstream of the magnet. An Allison type emittance rig is located in the LEBT to record transverse emittances before acceleration.

\section{RESULTS}

Both rf and beam tests have been successfully completed. The RFQ was operated in cw mode for all beam tests. The operation of the RFQ at peak voltage $(74 \mathrm{kV})$ is stable[4]. Beams of both $\mathrm{N}^{+}$and $\mathrm{N}_{2}^{+}$have been accelerated to test the RFQ at low and high power. Two beam emittances were used: a regular emittance beam of $\beta \epsilon_{x, y}=0.03 \pi \mathrm{mm}-\mathrm{mrad}$ and a collimated 'pencil' beam of $\beta \epsilon_{x, y}=0.003 \pi \mathrm{mm}$ mrad.

\subsection{Regular Emittance Beam}

Beam Capture Beam capture measurements as a function of RFQ vane voltage have been completed for each ion and for both unbunched and bunched input beams. The results are given in Fig. 2 (squares) along with efficiencies predicted by PARMTEQ (dashed lines). The RFQ capture efficiency at the nominal voltage is $80 \%$ in the bunched case (three harmonics) and 25\% for the unbunched case in reasonable agreement with predictions. The capture efficiencies for one harmonic and two harmonic pre-bunching are $63 \%$ and $74 \%$ respectively.

Energy and Energy Spread The energy of the beam as measured with the analyzing magnet is $55 \mathrm{keV} / \mathrm{u}$. The energy spread for the bunched and unbunched cases was measured at $\pm 0.4 \%$ and $\pm 0.7 \%$ respectively and compares well with PARMTEQ predictions (Fig. 3)

Transverse Emittance Transverse emittances were measured before and after the RFQ. The results show that 

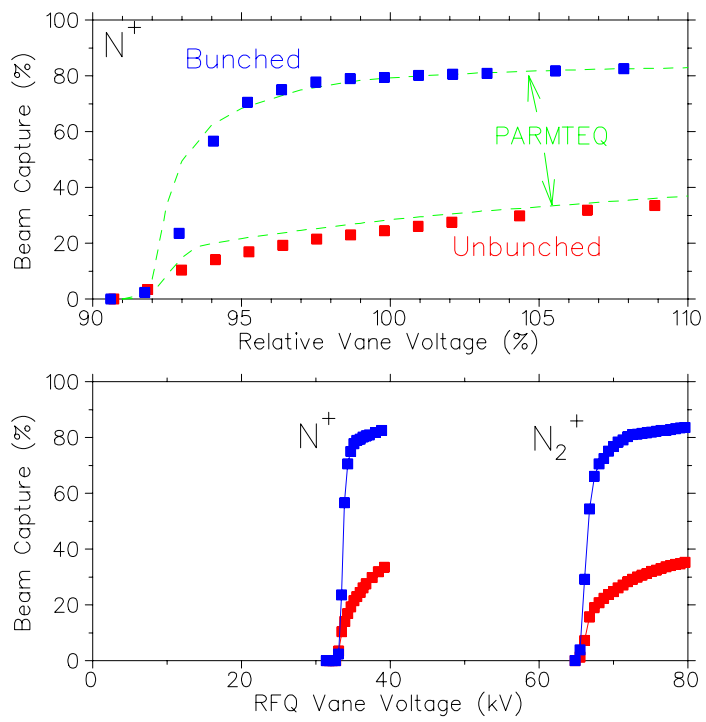

Figure 2: The beam capture (squares) for both bunched and unbunched initial beams of $\mathrm{N}^{+}$as a function of relative vane voltage are compared with PARMTEQ calculations (dashed lines). In (b) results for both $\mathrm{N}_{2}^{+}$and $\mathrm{N}^{+}$are plotted with respect to absolute vane voltage.
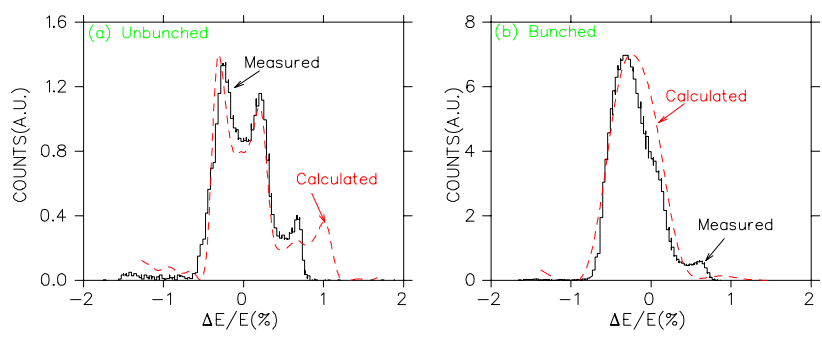

Figure 3: Results of energy spread measurements of accelerated $\mathrm{N}^{+}$beams for both (a) unbunched and (b) bunched cases. PARMTEQ simulation results are plotted for comparison.

when the matching is optimized the emittance growth in both planes is less than $10 \%$ for an initial beam of $15 \pi \mathrm{mm}$ mrad. A summary of the results for $\mathrm{N}^{+}$is presented in Fig. 4. Space charge forces limit the longitudinal acceptance for currents above $\sim 1 \mu \mathrm{A}$ but do not impact the transverse emittance.

\subsection{Pencil Beam Experiments}

During initial beam commissioning it was observed that when the system was tuned for maximum capture the beams emerged far off-center. There was concern that a dipole component internal to the RFQ was steering the beam. Two circular apertures of $2 \mathrm{~mm}$ separated by $0.7 \mathrm{~m}$ were placed in the RFQ injection line to create a centered 'pencil beam' to diagnose the problem. A vertical deflecting steerer was available downstream of the collimators to move the beam around the RFQ aperture.

The tests showed that the RFQ dipole component was negligible. However we found that the acceptance of the
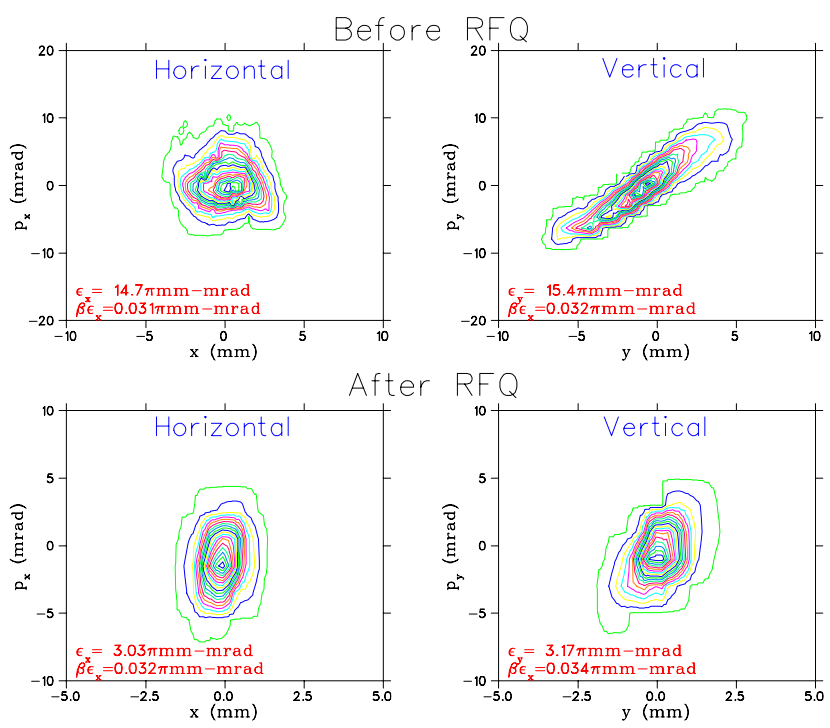

Figure 4: Transverse emittance measurements (4RMS values) for $\mathrm{N}_{14}$ before and after the RFQ. The normalized emittance values are also displayed.

RFQ is larger for off-centered beams. The two-term potential function yields the spatial component of the accelerating field as

$$
E_{z}=\frac{k A V}{2} I_{o}(k r) \sin k z
$$

where $k=2 \pi / \beta \lambda$ and $\beta$ is the relative particle velocity. Hence off-axis particles experience stronger fields than on-axis particles, particularly at injection where $\beta$ is small. In the ISAC RFQ the design synchronous phase is fixed at $-25^{\circ}$ at the nominal voltage but for off-axis particles the effective synchronous phase decreases, increasing the acceptance. A typical measure of the synchronous phase is the cut-off voltage, that voltage below which no acceleration can occur. Beam transmission as a function of RFQ voltage measured for a centered beam and two off-centered cases $\left(A_{c}=1.7 \mathrm{~mm}\right.$ and $A_{c}=2.7 \mathrm{~mm}$ ), plotted in Fig. 5, clearly shows that the cut-off voltage decreases for increasing amounts of off-centering. As the voltage nears cut-off the difference in acceptances between on-axis and off-axis particles is enhanced.

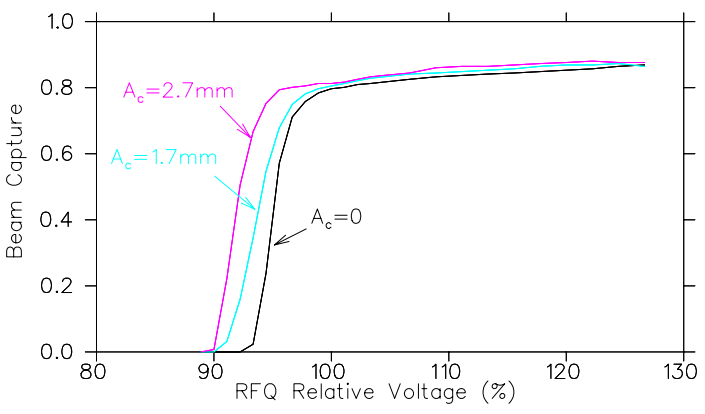

Figure 5: Transmission of 'pencil beam' as a function of RFQ voltage for a centered beam and two cases of offcentered beam. 
Transverse Acceptance and Centering Error An off-center small emittance beam passing through the RFQ makes coherent oscillations about the RFQ axis. The transverse phase advance per unit length is dependent on the RFQ voltage. The centering error can be determined by scanning the RFQ voltage to produce a $2 \pi$ change in the transverse phase advance while recording the final beam position. The rate of phase change with RFQ voltage, when compared with beam simulations, can be used to determine if the centering error was due to a mis-alignment at injection or was developed during acceleration from a dipole error. Beam position as a function of RFQ voltage is given in Fig. 6 for steering plate settings of 0, $400 \mathrm{~V}$ and $600 \mathrm{~V}$. The two deflected cases correspond to the transmission data plotted in Fig. 5. Based on the steering/transmission data the transverse acceptance was estimated to be $\geq 140 \pi \mathrm{mm}$ mrad.

Longitudinal Acceptance The longitudinal acceptance was measured for a centered and an off-centered beam $\left(A_{c}=2.7 \mathrm{~mm}\right)$ at the nominal RFQ voltage. The energy of the injected beam was varied at the source. For each energy step the phase of the bunched beam was varied with respect to the RFQ phase and the capture efficiency was recorded. Comparison of calculated and measured beam transmission contours for centered and off-centered cases are presented in Fig. 7. The settings where the capture dropped to $50 \%$ of peak value is used to define the longitudinal acceptance contour. The measured acceptance is estimated to be $220 \pi \%$-deg and $460 \pi \%$-deg at $35 \mathrm{MHz}$ for the centered and off-centered beams repectively $(0.35$ and $0.73 \pi \mathrm{keV} / \mathrm{u}-\mathrm{ns})$. PARMTEQ simulations predict corresponding acceptances of $310 \pi \%$-deg and $570 \pi \%$-deg (0.49 and $0.91 \pi \mathrm{keV} / \mathrm{u}-\mathrm{ns})$.

\section{TUNING AND MATCHING}

The increase in accelerating field strength for off-axis particles provides a large stable saddle point in the longitudinal motion. One problem however is that the transmission improves (at the expense of beam quality) as the beam moves off-center making tuning difficult. Larger unmatched beams are subject to a spread in the radial tune due

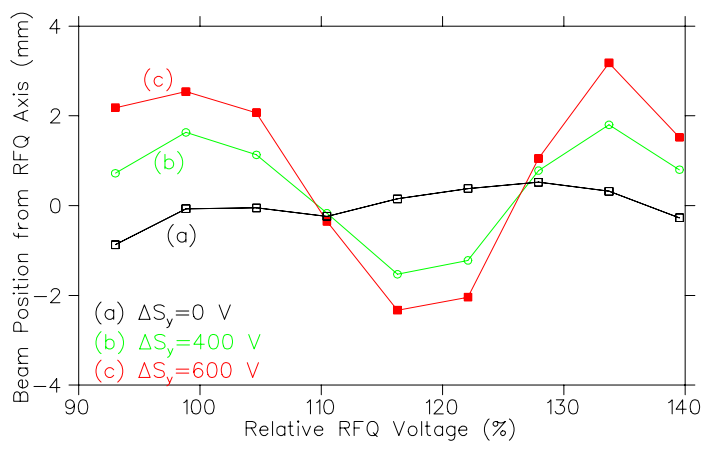

Figure 6: Beam position at RFQ exit as a function of RFQ voltage for steering plate strengths of $0,400 \mathrm{~V}$ and $600 \mathrm{~V}$.

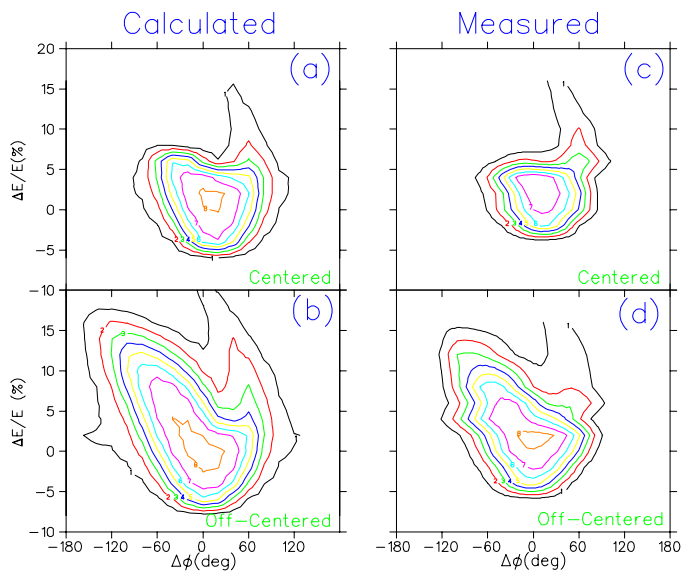

Figure 7: Beam transmission contours (plotted every 10\%) for calculated values ((a)centered and (b)off-centered) and measured values ((c)centered and (d)off-centered). Offcentered beams have a coherent amplitude of $A_{c}=2.7 \mathrm{~mm}$.

to the radial variation in the field strength and produce transverse emittance growth. A larger phase acceptance leads to a higher population of the tails in the accelerated longitudinal emittance. An optimization procedure was developed where the LEBT is tuned at high RFQ voltage for maximum transmission then the last steering plates and matching quadrupoles are re-tuned at an RFQ voltage near cut-off to achieve a local minimum in the transmission. The procedure results in both a centered and a matched beam since a matched beam sees more uniform accelerating fields on average.

\section{CONCLUSION}

The beam test results demonstrate a strong confirmation of both the beam dynamics design and the engineering concept and realization and give us full confidence in proceeding with the installation of the remaining twelve split rings. The 'pencil beam' proved invaluable in diagnosing the effects of off-center beams on beam capture.

\section{ACKNOWLEDGMENTS}

The tests were made possible by the collaborative effort of many groups; most notably the rf, LEBT, diagnostics and controls groups. Special thanks go to R. Poirier and W. Rawnsley. Thanks also to S. Koscielniak for useful discussions.

\section{REFERENCES}

[1] P. Schmor, "Initial Commissioning of the ISAC Radioactive Beam Facility", these proceedings.

[2] R. Poirier, "RF Systems of the TRIUMF ISAC Facility", these proceedings.

[3] S. Koscielniak, et al, "Beam Dynamics Studies on the ISAC RFQ at TRIUMF", Proceedings of the 1997 Part. Acc. Conf., Vancouver, 1997.

[4] R. Poirier, et al, "RF Tests on the Initial $2.8 \mathrm{~m}$ of the $8 \mathrm{~m}$ Long ISAC RFQ at TRIUMF”, Proceedings of LINAC98. 